\title{
Development of a novel HPTLC-based method for the simultaneous quantification of clinically relevant lipids from cells and tissue extracts
}

Michelle Pinault ${ }^{1}$, Cyrille Guimaraes ${ }^{1}$, Céline Ben Hassen ${ }^{1}$, Jorge L. Gutierrez-Pajares ${ }^{1}$, Stéphan Chevalier ${ }^{1}$, Caroline Goupille ${ }^{2}$, Pierre Bernard-Savary ${ }^{3}$, and Philippe G. Frank ${ }^{1}$ *

${ }^{1}$ Université de Tours, INSERM, UMR1069, Nutrition, Croissance et Cancer, Tours, France.

${ }^{2}$ CHRU Hôpital Bretonneau, Tours

${ }^{3}$ Chromacim, 170 Rue de Chatagnon, 38430 Moirans.

*Corresponding author: Philippe G. Frank, Université de Tours, INSERM, UMR1069, Nutrition, Croissance et Cancer, Tours, France.

E-mail:Philippe.Frank@univ-tours.fr

Keywords : Cholesterol, Triglycerides, Fatty Acids, Cancer 


\begin{abstract}
Lipids such as cholesterol, triglycerides, and fatty acids play important roles in the regulation of cellular metabolism and cellular signaling pathways and, as a consequence, in the development of various diseases. It is therefore important to understand how their metabolism is regulated to better define the components involved in the development of various human diseases. In the present work, we described the development and validation of an HPTLC method allowing the separation and quantification of free cholesterol, cholesteryl esters, nonesterified fatty acids, and triglycerides. This method will be of interest as the quantification of these lipids in one single assay is difficult to perform.
\end{abstract}




\section{Introduction}

Abnormality in the metabolism of lipids such as cholesterol (free or esterified), non-esterified fatty acids, and triglycerides have been shown to control the development of various diseases including atherosclerosis, obesity, cancer, nonalcoholic fatty liver disease, as well as other metabolic diseases. [1-4]. In cancer, they have been demonstrated to play an important role in the regulation of cancer progression. Their functions include the regulation of cellular signaling pathways involved in cell cycle regulation as well as other pathways regulating cancer cell migration and survival [5-7]. In several types of cancer, plasma cholesterol levels have been shown to regulate tumor formation and cancer aggressiveness [8,9]. In vascular disease, studies have now demonstrated the role of cholesterol and triglycerides in the development of atherosclerosis [10]. After accumulation in the form of LDL-derived cholesterol in the arterial wall of blood vessels, an inflammatory reaction leads to the attraction of monocyte/macrophages in the intima and eventually atheroma development [11]. Finally, obesity, which is characterized by increased adipose tissue and increased triglyceride accumulation, is associated with defect in the metabolism of many lipids such as cholesterol and fatty acids, and its appearance is associated with the development of other diseases such as cardiovascular disease, type 2 diabetes, and cancer [12].

Taken together, these data suggest that a method that could facilitate the simultaneous cellular or tissue quantification of these lipids and allow valid comparisons between samples would be invaluable to study the regulation and the role of these lipids in human diseases. In the present study, we described the development of a method for the reproducible quantification of these lipids (Fig. 1) in cells and tissue extracts. 


\section{Materials and methods}

\section{Reagents}

Free cholesterol (FC), cholesteryl oleate (CE), oleic acid (NEFA) and triolein (TG) were obtained from Sigma-Aldrich (Saint-Quentin Fallavier, France). All other reagents were analytical grade.

Phospho-vanillin was prepared as followed: $0.99 \mathrm{~g}$ of Vanillin (Sigma-Aldrich) was dissolved in $10 \mathrm{~mL}$ ethanol $100 \%$ before dilution into $153 \mathrm{~mL}$ with distilled water. This solution was mixed with $337 \mathrm{~mL}$ of $85 \% \mathrm{H}_{3} \mathrm{PO}_{4}(\mathrm{w} / \mathrm{v})$ with constant stirring. The resulting solution was prepared before each use and kept in a dark bottle at room temperature. Perchloric acid (65\%) was obtained from Sigma-Aldrich.

\section{HPTLC instruments and chromatographic conditions}

Standard calibration curves were obtained by spotting standards of known lipid composition. For sample analyses, after lipid extraction with the method from Bligh and Dyer [13], lipid extracts were also spotted on HPTLC precoated silica gel glass plates $60 \mathrm{~F}_{254}(20 \times 10 \mathrm{~cm}$; 1.05642.0001; E. Merck, Darmstadt, Germany) using a Camag autosampler ATS4 sample applicator (Camag, Muttenz Switzerland) equipped with a $25-\mu \mathrm{L}$ dosing syringe. For each plate, 14 tracks were used, five were used for calibration standards and nine for sample application. In successive plates, calibration standards were spotted on different tracks to avoid side effects.

Before each experiment, plates were pre-developed once in chloroform-methanol 1:1 (v/v), air-dried, and activated at $110^{\circ} \mathrm{C}$ in an oven for $20 \mathrm{~min}$. Samples were applied in the form of $10-\mathrm{mm}$ band shapes, $1.5 \mathrm{~cm}$ from the bottom of the Silica plate at a constant flow rate of 150 $\mathrm{nL} / \mathrm{s}$. under nitrogen flow. This spray-on technique and the flow rate adjusted to the type of sample solvent to be applied, dries out the solvent during the application. It avoids diffusion and permits an optimal sample band focus that allows a good separation and a homogenous quantification. For development, the mobile phase used was a mixture of hexane:ether:acetic acid (70:30:1; v/v/v). Linear ascending development was carried out in a 20x10cm twin trough glass chamber (Camag, Muttenz, Switzerland) pre-equilibrated with the mobile phase. Optimal chamber saturation time for the mobile phase was $15 \mathrm{~min}$ at room temperature without saturation paper. The length of development was $10 \mathrm{~cm}$. We used $20 \mathrm{~mL}$ of mobile phase for saturation and migration $(10 \mathrm{~mL}$ in the tank facing the plate and $10 \mathrm{~mL}$ in the tank where the plate is developed). Subsequent to the development, HPTLC plates were air-dried 
under a ventilated hood for 90 min after migration. Dried plates were treated by dipping for 1 min in an immersion tank containing a $50 \%$ perchloric acid solution. After a 2-hour drying period at room temperature, plates were placed on a plate heater (TLC Plate Heater 3, Camag Muttenz Switzerland) for $16 \mathrm{~min}$ at $160^{\circ} \mathrm{C}$ to allow carbonization for the staining reaction. Densitometric analysis was performed using a TLC/HPTLC video-densitometer (TLC Visualizer 2 Camag, with Videoscan software, Camag, Muttenz, Switzerland).

\section{Standard solution preparation}

To establish the assay range, the limit of detection was determined for all applications. We tested concentrations ranging from $0.04 \mathrm{mg} / \mathrm{ml}$ to $0.8 \mathrm{mg} / \mathrm{ml}$. A standard solution at $1 \mathrm{mg} / \mathrm{mL}$ was prepared and used for the calibration curve. For each authentic standard of lipid, we performed a calibration curve with 5 points at increasing levels to quantify lipids on the plates. Stock solutions and standards were aliquoted and kept in glass tubes (with a screw cap and silicone/PTFE seal) at $-20^{\circ} \mathrm{C}$. Under these conditions, products were stable for one year.

\section{Method validation}

The method was validated as described by the ICH harmonization guidelines [14] in terms of linearity, accuracy, specificity, repeatability, system repeatability, and intermediate precision of measurement for peak area.

The percent of recovery was calculated by using the formula, \% recovery $=[(\mathrm{T}-\mathrm{A}) / \mathrm{S}] \times 100$, where: $\mathrm{T}$ is the percentage added to the maximum value in range (i.e. 50, 100, 150, and $200 \%), \mathrm{S}$ is the maximum value in range, $\mathrm{A}$ is the difference between the area at the considered percentage and the area of S. Recovery studies were carried out by spiking the stock solutions ( $1 \mathrm{mg} / \mathrm{mL})$ with $50 \%, 100 \%, 150 \%$, and $200 \%$ of the standard lipid solution. After dilutions were made, recovery studies were performed. Precision of the measurements was verified by repeatedly testing $(n=10)$ each mixed standard solutions (five different concentrations) containing all the lipids. Experiments were repeated the same day using several HPTLC plates.

LOD and LOQ were calculated from the following equations: $\mathrm{LOD}=3 \times(\mathrm{SD} / \mathrm{S})$ and $\mathrm{LOQ}=10 \times(\mathrm{SD} / \mathrm{S})$, with $\mathrm{S}$, slope of the calibration curve and $\mathrm{SD}$, standard deviation of the blank signal ( $\mathrm{n}=10$ measurements on different plates). For this determination, mean blank was obtained for each lipid class using a blank lane by spotting the solvent (chloroform/methanol, $2: 1, \mathrm{v} / \mathrm{v}$ ) and reading the signal at the same retention factor (Rf) as that of the corresponding lipids. 
The limit of quantification (LOQ) was also estimated using the Eurachem approach [15]. Five concentration close to the quantification point were used, and the relative standard deviation was plotted against the measured concentration. The corresponding limit of quantification was obtained from this graph for a relative standard deviation of $10 \%$.

Statistical analyses were performed with StatPlus 6.03.

\section{Spectrophotometric quantification of lipids using phospho-vanillin}

The sulfo-phospho-vanillin assay [16] was performed to determine the accuracy of our method. Briefly, two $\mathrm{mL}$ of concentrated $\mathrm{H}_{2} \mathrm{SO}_{4}(98 \%$ w/v solution) were added to standards at different concentrations after solvent evaporation. Tubes were heated for $20 \mathrm{~min}$ in a boiling water bath and cooled down for $4 \mathrm{~min}$. To each tube, $4 \mathrm{~mL}$ of the phospho-vanillin reagent were added for color development. After a 15-minute cooling period at $25{ }^{\circ} \mathrm{C}$, absorbance was read on a Secoman-S1000 spectrophotometer.

\section{Cell culture}

The MCF-10A cell line was obtained from the American Tissue Culture Collection (ATCC) (Molsheim, France). The MDA-MB-231 and MCF-7 cell lines were obtained from Cell biolabs, Inc. (San Diego, CA) and GenTarget (San Diego, CA), respectively. MCF-10A cells were grown DMEM /F12 Ham's Mixture supplemented with 5\% horse serum (SigmaAldrich), EGF (20 ng/ml, Sigma-Aldrich), insulin (10 $\mathrm{g} / \mathrm{ml}$, Sigma- Aldrich), hydrocortisone (0.5 mg/ml, Sigma- Aldrich), cholera toxin (100 ng/ml, Sigma-Aldrich), 100 units/ml penicillin and $100 \mu \mathrm{g} / \mathrm{ml}$ streptomycin. The other cell lines were grown in Dulbecco's modified Eagle's media (DMEM) containing 10\% Fetal Bovine Serum (FBS) and 100 units $/ \mathrm{ml}$ penicillin and $100 \mu \mathrm{g} / \mathrm{ml}$ streptomycin. Cells were placed in a humidified incubator kept at $37^{\circ} \mathrm{C}$ with $5 \% \mathrm{CO}_{2}$. For lipid extraction, cells were grown in T175 flash to 90-95\% confluence and were washed with PBS. They were harvested using a cell scraper. Lipids from the cell pellets were extracted using the method Bligh and Dyer [13].

For fluorescent microscopy, cells were seeded on coverslips and incubated in complete media. After 1 day in culture, cells were fixed in $2 \%$ paraformaldehyde, incubated with $1 \%$ BSA in PBS, and incubated with $300 \mathrm{nM}$ Nile red (in PBS containing calcium and magnesium (CM)). After several wash in PBS-CM cells were mounted using SlowFade ${ }^{\circledR}$ diamond antifade reagent containing the nuclear stain DAPI (Fischer Scientific). Cells were observed on a Nikon TI-S microscope and analyzed using both NIS-BR software (Nikon, France). 


\section{Results and Discussion}

The method presented in this study was directly adapted from a TLC method previously used in our laboratory to separate fatty acids from triglycerides [17]. We tested it to determine whether we could simultaneously separate, detect, and quantify free cholesterol, nonesterified fatty acids, triglycerides, and cholesteryl esters (ig. 1).

\section{Calibration curves - Linearity and range}

A mixture of chloroform and methanol $(2: 1, \mathrm{v} / \mathrm{v})$ was used to prepare solutions for all lipids. Using an automated sample applicator, spots were made for each range of mixtures containing free cholesterol, non-esterified fatty acids, triglycerides, and cholesteryl esters at increasing concentrations. A range of concentrations (each one repeated 10 times) was loaded onto each plate. Plates after run and the resulting chromatograms (example presented Fig. 2) allowed us to obtain the calibration curves presented in Fig. 3. All lipids were well separated and formed identifiable bands.

Images of plates with standards were transferred to the Videoscan software. After densitometric analysis, calibration curves were obtained by plotting the peak area under the curve against the concentration of standards. Standard curves were obtained for each of the analyzed lipids. Table 1 shows Rf values obtained for free cholesterol, non-esterified fatty acids, triglycerides and cholesteryl esters. In all cases, linearity was observed at levels ranging from:

- 1000 to $8000 \mathrm{ng} / \mathrm{spot}$ for free cholesterol (FC).

- 2000 to $8000 \mathrm{ng} / \mathrm{spot}$ for non-esterified fatty acids (NEFA).

- 2000 to $8000 \mathrm{ng} / \mathrm{spot}$ for triglycerides (TG).

- 1000 to $8000 \mathrm{ng} / \mathrm{spot}$ for cholesteryl esters (CE).

Correlation coefficients $\left(r^{2}\right)$ are presented in $\underline{\text { Table } 2}$ for the calibration curves shown in Fig. 3. Linearity was confirmed using 10 measurements per concentration. Fives different plates were used.

\section{Accuracy of the Method}

We compared our technique with the method of Knight et al, [16] originally used to quantify plasma lipids levels. This method was first adapted for oleic acid and compared to the original work of Knight et al. (ig. 4). A small relative deviation of 1.6\%, was observed. In a separate experiment, concentration ranges of triglycerides, non-esterified fatty acids, free cholesterol 
and cholesteryl esters were also analyzed with this spectrophotometric method (Fig. 5). The aim of this analysis was to compare the two methods ( $\underline{\text { Table 3 }}$ ). In all cases, the HPTLC method presented in this work had a sensitivity comparable to that of the spectrophotometric analysis. However, our method allows for a broader range of lipid analysis than the sulfophospho vanillin assay, which is not lipid specific.

The accuracy of the method was also established using the recovery technique (i.e., external standard addition method). A known amount of standard was added at four different levels to a pre-analyzed sample. Each determination was performed three times. The results of the recovery study presented in $\underline{\text { Table } 4}$ were found to be within acceptable limits (value $\pm 10 \%$ ).

\section{Precision}

Repeatability and intermediate precision were determined at different concentrations levels. The percent relative standard deviation (\%RSD) values for repeatability and intermediate precision as shown in Table $\mathbf{5}$ reveal that the proposed method is precise.

\section{Limit of detection (LOD)and limit of quantification (LOQ)}

Limit of detection (LOD) and limit of quantification (LOQ) values are presented in Table 6. This method is therefore sensitive for our analyses. Importantly, the LOQ values determined as described in the Material and Method section ( $\underline{\text { Table 6) }}$ ) are comparable to those derived from the method using the Eurachem approach (Fig. 6) [15].

\section{Specificity and Selectivity}

Specificity of the method was assessed by analyzing authentic lipid standards. Identity of the lipids present in three different mammary epithelial cell lines (MCF-10A, MCF-7, and MDAMB-231; after Bligh and Dyer extraction [13]) was determined by comparison of the lipids' Rf with values obtained for authentic lipid standards (Fig. 7). All these cell lines contain triglycerides, non-esterified fatty acids, cholesteryl esters, and free cholesterol. This assay could allow us to determine the effect of a specific treatment on lipid metabolism (nonesterified fatty acids, free cholesterol, cholesteryl esters, triglycerides) using these cell lines. Interestingly, the MCF-7 cell line contains significantly less EC compared to the non cancerous MCF-10A cell line and the more aggressive MDA-MB-231 cell lines. FC, NEFA, and TG levels were not significantly different. The significance of the increased EC content observed in MCF-10A and MDA-MB231 cells remains to be determined. Importantly, these 
findings were confirmed by fluorescent microscopy using the neutral lipid stain, Nile Red (Fig. 8).

Finally, other related lipid species did not have the same Rf as those studied in the present work. Diacylglycerol and cardiolipin had Rf of 0.28 and 0.01 , respectively. Importantly, all types of phospholipid remain at the origin.

\section{Statistical analysis}

For these lipid determinations, variance homogeneity of our data standard curve was verified using the Cochran's C test for homoscedasticity ( $\underline{\text { Table 6)}}$. Furthermore, to validate the linear regression model and test if the slope was different from 0, a Fischer's test was applied. The value obtained for each lipid type was compared with the values from the F table. For this study, the test was accepted for all lipids at $p<0.05$. Our accuracy results show that the values obtained by HPTLC are significantly correlated with the amounts of lipids used in each case ( $\underline{\text { Table 6)}}$. It therefore validates the use of our method for the quantification of these lipids in plasma, tissue, or cell extracts.

\section{Conclusions}

The present study demonstrates that our HPTLC-densitometric analysis method is reproducible, repeatable, selective, and accurate for the analysis of the different lipids examined. Moreover, this technique is simple, fast, robust, and less expensive than HPLC. Multiple detection methods can be used in this context: UV (254 or $366 \mathrm{~nm}$ ) or white light after derivatization using various reagents. However, we found that the system used in the present work gave the best results. Another important advantage is that several samples can be analyzed at once, thereby limiting the use of solvent and accelerating the analyses. Moreover, this method may allow us to quantify free cholesterol, cholesteryl esters, non-esterified fatty acids, and triglycerides present in plasma, adipose tissues, or other tissue/cell extracts containing these types of lipids. As an example, we show that this HPTLC-densitometry method can be routinely used for the quantification of these lipid classes in cancer cells. 
Acknowledgements: This work was supported by the Faculty of Medicine at the University of Tours. JLGP was supported by Le Studium (Région Centre-Val de Loire, France). PGF was supported by grants from INCa PLBio (2018-145), the Lipids ARD2020-Biodrug project (Région Centre-Val de Loire, France), La Ligue contre le Cancer (Indre et Loire, Loir et Cher, and Vienne), by an Academic Research Grant from the Région Centre-Val de Loire (France) and by the Canceropole Grand-Ouest (Mature project). We thank Dr. Jacques Potier, Violetta Guerin, and Simon Bergerard for technical support.

Conflicts of Interest: The authors declare that they have no conflict of interest. 


\section{References}

1. Radner FP, and Fischer J (2014) The important role of epidermal triacylglycerol metabolism for maintenance of the skin permeability barrier function. Biochim Biophys Acta $1841: 409-415$

2. Walenbergh SM, and Shiri-Sverdlov R (2015) Cholesterol is a significant risk factor for non-alcoholic steatohepatitis. Expert Rev Gastroenterol Hepatol 9 : 1343-1346

3. Yang X, Lee HM, and Chan JC (2015) Drug-subphenotype interactions for cancer in type 2 diabetes mellitus. Nat Rev Endocrinol $11: 372-379$

4. Perona JS (2017) Membrane lipid alterations in the metabolic syndrome and the role of dietary oils. Biochim Biophys Acta 1859 : 1690-1703

5. Gutierrez-Pajares JL, Ben Hassen C, Chevalier S, and Frank PG (2016) SR-BI: Linking Cholesterol and Lipoprotein Metabolism with Breast and Prostate Cancer. Front Pharmacol $7: 338$

6. Voisin M, de Medina P, Mallinger A, Dalenc F, Huc-Claustre E, Leignadier J, Serhan N, Soules R, Segala G, Mougel A, Noguer E, Mhamdi L, Bacquie E, Iuliano L, Zerbinati C, Lacroix-Triki M, Chaltiel L, Filleron T, Cavailles V, Al Saati T, Rochaix P, Duprez-Paumier R, Franchet C, Ligat L, Lopez F, Record M, Poirot M, and Silvente-Poirot $S$ (2017) Identification of a tumor-promoter cholesterol metabolite in human breast cancers acting through the glucocorticoid receptor. Proc Natl Acad Sci U S A 114 : E9346-E9355

7. Figiel S, Pinault M, Domingo I, Guimaraes C, Guibon R, Besson P, Tavernier E, Blanchet P, Multigner L, Bruyere F, Haillot O, Mathieu R, Vincendeau S, RiouxLeclercq N, Lebdai S, Azzouzi AR, Perrouin-Verbe MA, Fournier G, Doucet L, Rigaud J, Renaudin K, Maheo K, and Fromont G (2018) Fatty acid profile in periprostatic adipose tissue and prostate cancer aggressiveness in African-Caribbean and Caucasian patients. Eur J Cancer 91 : 107-115

8. Llaverias G, Danilo C, Mercier I, Daumer K, Capozza F, Williams TM, Sotgia F, Lisanti MP, and Frank PG (2011) Role of cholesterol in the development and progression of breast cancer. Am J Pathol 178 : 402-412

9. Llaverias G, Danilo C, Wang Y, Witkiewicz AK, Daumer K, Lisanti MP, and Frank PG (2010) A Western-type diet accelerates tumor progression in an autochthonous mouse model of prostate cancer. Am J Pathol 177 : 3180-3191

10. Goldberg IJ (2018) 2017 George Lyman Duff Memorial Lecture: Fat in the Blood, Fat in the Artery, Fat in the Heart: Triglyceride in Physiology and Disease. Arterioscler Thromb Vasc Biol 38 : 700-706

11. Glass CK, and Witztum JL (2001) Atherosclerosis. the road ahead. Cell 104 : 503-516

12. Ben-Shmuel S, Rostoker R, Scheinman EJ, and LeRoith D (2016) Metabolic Syndrome, Type 2 Diabetes, and Cancer: Epidemiology and Potential Mechanisms. Handb Exp Pharmacol 233 : 355-372

13. Bligh EG, and Dyer WJ (1959) A rapid method of total lipid extraction and purification. Can J Biochem Physiol 37 : 911-917

14. Guideline IHT (2005 Q2 (R1)) Validation of analytical procedures: text and methodology. International Conference on Harmonisation of Technical Requirements for Registration of Pharmaceuticals for Human Use

15. Spangenberg B, Poole CF, and Weins C (2010) Planning an Analysis and Validation in TLC. Quantitative Thin-Layer Chromatography, Springer: Berlin, Heidelberg

16. Knight JA, Anderson S, and Rawle JM (1972) Chemical basis of the sulfo-phosphovanillin reaction for estimating total serum lipids. Clin Chem $18: 199-202$ 
17. Maillot F, Garrigue MA, Pinault M, Objois M, Theret V, Lamisse F, Hoinard C, Antoine JM, Lairon D, and Couet C (2005) Changes in plasma triacylglycerol concentrations after sequential lunch and dinner in healthy subjects. Diabetes Metab $31: 69-77$ 


\section{Tables}

Table 1: HPTLC profile of a standard lipid solution

\begin{tabular}{|c|c|c|c|c|c|c|c|c|c|}
\hline \multirow{2}{*}{ Lipid } & \multicolumn{2}{|c|}{ Peak Beginning } & \multicolumn{3}{c|}{ Peak Maximum } & \multicolumn{2}{c|}{ Peak End } & \multicolumn{2}{c|}{ Peak Area } \\
\cline { 2 - 10 } & Rf & H & Rf & H & {$[\%]$} & Rf & H & A & {$[\%]$} \\
\hline CE & 0.828 & 0.0 & 0.881 & 2121.8 & 30.05 & 0.897 & 0.0 & 24292.9 & 28.81 \\
\hline TG & 0.769 & 0.0 & 0.800 & 646.1 & 9.15 & 0.825 & 9.8 & 7998.5 & 9.49 \\
\hline NEFA & 0.359 & 26.1 & 0.413 & 506.8 & 7.18 & 0.473 & 19.1 & 9861.1 & 11.69 \\
\hline FC & 0.179 & 210.8 & 0.203 & 3786.8 & 53.63 & 0.228 & 293.8 & 42168.7 & 50.01 \\
\hline
\end{tabular}

Rf: retention factor, ratio distance migrated / total distance covered by the solvent.

$\mathrm{H}$ : peak height.

These values were obtained with $8 \mu \mathrm{g} / \mathrm{spot}$ for each lipid.

FC: free cholesterol, NEFA: non-esterified fatty acids, TG: triglycerides, CE: cholesteryl esters. 
Table 2: Linear regression data obtained for the different calibration curves

\begin{tabular}{|c|c|c|c|}
\hline Lipid & Slope $( \pm \boldsymbol{R S D})$ & Correlation coefficient & $\begin{array}{c}\text { Equation of the regression line } \\
\text { (peak surface =slope } \mathbf{x} \text { amount } \\
\text { deposited) }\end{array}$ \\
\hline FC & $28590( \pm 3622)$ & 0.9959 & $\mathrm{y}=28590 \mathrm{x}+1605$ \\
\hline NEFA & $9182( \pm 1284)$ & 0.9952 & $\mathrm{y}=9182 \mathrm{x}+49$ \\
\hline TG & $7969( \pm 1371)$ & 0.9933 & $\mathrm{y}=7969 \mathrm{x}-1300$ \\
\hline CE & $26657( \pm 4621)$ & 0.9940 & $\mathrm{y}=26657 \mathrm{x}+510$ \\
\hline
\end{tabular}

FC: free cholesterol, NEFA: non-esterified fatty acids, TG: triglycerides, CE: cholesteryl esters. 
Table 3: Comparison of the lipid analyses performed by HPTLC and using the spectrophotometer method

\begin{tabular}{|l|c|c|l|l|c|}
\hline \multicolumn{3}{|c|}{ HPTLC Method } & \multicolumn{3}{c|}{ Spectrophotometry method } \\
\hline \multicolumn{1}{|c|}{ Lipid } & Concentration & Sensitivity & \multicolumn{1}{c|}{ Lipid } & Concentration & Sensitivity \\
\hline TG & $0.04 \mathrm{mg} / \mathrm{mL}$ & $0.40 \mu \mathrm{g}$ & TG & $0.04 \mathrm{mg} / \mathrm{mL}$ & $0.36 \mu \mathrm{g}$ \\
\hline NEFA & $0.05 \mathrm{mg} / \mathrm{mL}$ & $0,50 \mu \mathrm{g}$ & NEFA & $0.05 \mathrm{mg} / \mathrm{mL}$ & $1.29 \mu \mathrm{g}$ \\
\hline FC & $0.05 \mathrm{mg} / \mathrm{mL}$ & $0,55 \mu \mathrm{g}$ & FC & $0.05 \mathrm{mg} / \mathrm{mL}$ & $0.93 \mu \mathrm{g}$ \\
\hline CE & $0.04 \mathrm{mg} / \mathrm{mL}$ & $0,63 \mu \mathrm{g}$ & CE & $0.04 \mathrm{mg} / \mathrm{mL}$ & $0,77 \mu \mathrm{g}$ \\
\hline
\end{tabular}

FC: free cholesterol, NEFA: non-esterified fatty acids, TG: triglycerides, CE: cholesteryl esters. 
Table 4: Recovery studies of the different lipids after $50 \%, 100 \%$ and $150 \%$ addition of the standard lipid solution

\begin{tabular}{|c|c|c|c|c|c|c|c|}
\hline Lipid & \begin{tabular}{|c|}
$\begin{array}{c}\text { Amount } \\
\text { at } \\
100 \% \\
(\mu \mathrm{g})\end{array}$ \\
\end{tabular} & Levels & $\begin{array}{c}\text { Expected amount } \\
\text { to be assayed } \\
(\mu \mathrm{g})\end{array}$ & $\begin{array}{c}\text { Area observed } \\
\text { (AU) }\end{array}$ & $\begin{array}{c}\text { Amount } \\
\text { calculated } \\
(\mu \mathrm{g})\end{array}$ & $\begin{array}{c}\text { Recovery (\% mean } \\
\pm \mathrm{SD})\end{array}$ & $\mathrm{n}$ \\
\hline \multirow{4}{*}{$\mathrm{FC}$} & \multirow{4}{*}{4} & $50 \%$ & 2 & 65084 & 1.99 & $99.00 \pm 1.02$ & 3 \\
\hline & & $100 \%$ & 4 & 130569 & 4.00 & $100.00 \pm 0.59$ & 3 \\
\hline & & $150 \%$ & 6 & 193568 & 5.93 & $98.90 \pm 0.72$ & 3 \\
\hline & & $200 \%$ & 8 & 253054 & 7.75 & $96.90 \pm 0.58$ & 3 \\
\hline \multirow{4}{*}{ NEFA } & \multirow{4}{*}{4} & $50 \%$ & 2 & 27574 & 1.98 & $99.00 \pm 1.05$ & 3 \\
\hline & & $100 \%$ & 4 & 55833 & 4.00 & $100.00 \pm 0.68$ & 3 \\
\hline & & $150 \%$ & 6 & 75504 & 5.41 & $90.00 \pm 1.10$ & 3 \\
\hline & & $200 \%$ & 8 & 106589 & 7.63 & $95.00 \pm 1.09$ & 3 \\
\hline \multirow{4}{*}{ TG } & \multirow{4}{*}{4} & $50 \%$ & 2 & 25092 & 1.97 & $99.00 \pm 0.57$ & 3 \\
\hline & & $100 \%$ & 4 & 50916 & 4.00 & $100.00 \pm 0.88$ & 3 \\
\hline & & $150 \%$ & 6 & 68614 & 5.39 & $90.00 \pm 0.96$ & 3 \\
\hline & & $200 \%$ & 8 & 99580 & 7.82 & $97.70 \pm 0.90$ & 3 \\
\hline \multirow{4}{*}{$\mathrm{CE}$} & \multirow{4}{*}{4} & $50 \%$ & 2 & 54984 & 1.82 & $91 \pm 0.77$ & 3 \\
\hline & & $100 \%$ & 4 & 120823 & 4 & $100 \pm 0.59$ & 3 \\
\hline & & $150 \%$ & 6 & 174019 & 5.76 & $96.00 \pm 1.01$ & 3 \\
\hline & & $200 \%$ & 8 & 237512 & 7.86 & $98.28 \pm 0.95$ & 3 \\
\hline
\end{tabular}

FC: free cholesterol, NEFA: non-esterified fatty acids, TG: triglycerides, CE: cholesteryl esters. 


\section{Table 5: Repeatability and intermediate precision data for the different lipids}

\begin{tabular}{|l|c|c|c|c|}
\hline Quantity $(\mu \mathrm{g})$ & \multicolumn{4}{|c|}{$\mathbf{2}$} \\
\hline Lipid & FC & NEFA & TG & CE \\
\hline Repeatability $(\%)$ & 7.48 & 10.01 & 8.49 & 8.16 \\
\hline $\begin{array}{l}\text { Intermediate precision } \\
(\%)\end{array}$ & 7.49 & 13.60 & 11.60 & 10.97 \\
\hline \multicolumn{5}{|c|}{} \\
\hline Quantity $(\mu \mathrm{g})$ & FC & NEFA & TG & CE \\
\hline Lipid & 6.20 & 7.44 & 11.46 & 8.96 \\
\hline Repeatability (\%) & 6.80 & 7.99 & 13.59 & 9.88 \\
\hline $\begin{array}{l}\text { Intermediate precision } \\
(\%)\end{array}$ & & & & \\
\hline
\end{tabular}

FC: free cholesterol, NEFA: non-esterified fatty acids, TG: triglycerides, CE: cholesteryl esters. $\mathrm{n}=10$ for each concentration and each lipid. 


\section{Table 6: Method validation parameters for the quantification of the different lipids}

\begin{tabular}{|l|c|c|c|c|}
\hline Lipid & FC & NEFA & TG & CE \\
\hline LOD (ng/spot) & 556 & 1746 & 1284 & 639 \\
\hline LOQ (ng/spot) & 913 & 3092 & 2596 & 1593 \\
\hline $\begin{array}{l}\text { Regression } \\
\text { coefficient }\end{array}$ & 0.9858 & 0.9991 & 0.9993 & 0.9987 \\
\hline $\begin{array}{l}\text { Cochran C }\left(\mathrm{C}_{\text {calc }} / \mathrm{C}_{\text {ref }},\right. \\
p<0.01)\end{array}$ & $0.4902 / 0.5702$ & $0.4520 / 0.5702$ & $0.3600 / 0.5702$ & $0.3343 / 0.5702$ \\
\hline
\end{tabular}

FC: free cholesterol, NEFA: non-esterified fatty acids, TG: triglycerides, CE: cholesteryl esters. 


\section{Figure legends}

Fig. 1: Lipid structure representations. a. Free cholesterol. b. Triglyceride. c. Cholesteryl ester. d. Non-esterified fatty acid. R1, R2, and R3 represent alkyl groups.

Fig. 2: Profile of a standard separation realized by HPTLC. Samples, in the form of 7-mm bands, were spotted at a constant application rate of $150 \mathrm{~nL} / \mathrm{s}$ under nitrogen (6 bars). For development, the mobile phase used was a mixture of hexane:ether:acetic acid (70:30:1; $\mathrm{v} / \mathrm{v} / \mathrm{v})$. Linear ascending development was carried out in a $20 \times 10 \mathrm{~cm}$ twin trough glass chamber (Camag, Muttenz, Switzerland) pre-equilibrated with the mobile phase. Optimal chamber saturation time for the mobile phase was $15 \mathrm{~min}$ at room temperature. The length of development was $7 \mathrm{~cm}$. Subsequent to the development, HPTLC plates were air-dried and then treated by immersion in a 50\% perchloric acid solution for 2 min. After a 2-hour drying period, plates were placed on a plate heater to allow carbonization. Lane 1 to 9: standards curves of each class of lipids. Amounts of standard loaded (lane number: $\mu \mathrm{g}$ ): $1: 1 ; 2: 2 ; 3: 3$; $4: 4 ; 5: 5 ; 6: 6 ; 7: 7 ; 8: 8$. Images of standards were transferred to the Videoscan software, and densitometric scan were obtained. The densitometric scan presented in this figure was obtained for the $8 \mu \mathrm{g}$ standards. FC: free cholesterol, NEFA: non-esterified fatty acids, TG: triglycerides, CE: cholesteryl esters.

Fig. 3: Calibration curves of the different lipids separated by HPTLC. Using an automated sample applicator, deposits were made for each range of mixtures containing free cholesterol, non-esterified fatty acids, triglycerides, and cholesteryl esters at increasing concentrations. Images of standards and samples were transferred to the Videoscan software (version 1.2), and calibration curves were obtained by plotting the peak area under the curve against the concentration of applied standards. Standard curves were obtained for all the analyzed lipids. FC: free cholesterol, NEFA: non-esterified fatty acids, TG: triglycerides, CE: cholesteryl esters.

Fig. 4: Comparison of the methods from Knight et al. [16] with the present one for the analysis of oleic acid. After spectrophotometric quantification of lipids using the phosphovanillin method [16], standard curves obtained for the newly developed method and the phospho-vanillin method were compared for oleic acid. 
Fig. 5: Quantification of the various lipids using the methods from Knight et al. After spectrophotometric quantification of lipids using the phospho-vanillin method [16], standard curves obtained with the phospho-vanillin method were plotted for free cholesterol, triglycerides, cholesteryl esters, and oleic acid. FC: free cholesterol, NEFA: non-esterified fatty acids, TG: triglycerides, CE: cholesteryl esters.

Fig. 6: Precision profile obtained for the different lipids. RSD values were determined for analyses using $1-8 \mu \mathrm{g}$ of FC and CE or $2-8 \mu \mathrm{g}$ of NEFA and TG. LOQ values are indicated for an RSD of $10 \%$.

Fig. 7: Analysis of a lipid extracts obtained from mammary cell lines. Cells were grown in DMEM $10 \%$ FBS. $10^{6}$ cells were collected and lipids were extracted by the method of Bligh and Dyer [13]. After extraction, lipids were separated by HPTLC as described in the Material and Methods section. After quantification, the composition of each cell line for the four different lipids was reported per mg of cell proteins.

Fig. 8: Analysis of a neutral lipid content in human mammary cell lines by fluorescence microscopy using Nile Red. Cells were seeded on coverslips and incubated in complete media. After 1 day in culture, cells were fixed in $2 \%$ paraformaldehyde, incubated with $1 \%$ BSA in PBS, and incubated with $300 \mathrm{nM}$ Nile red (in PBS containing calcium and magnesium (CM)). After several wash in PBS-CM cells were mounted using SlowFade ${ }^{\circledR}$ diamond antifade reagent containing DAPI. Cells were observed on a Nikon TI-S microscope and analyzed using both NIS-BR software (Nikon, France). Red: Nile red staining (neutral lipids); blue: DAPI staining (nuclear stain). 
Figure 1

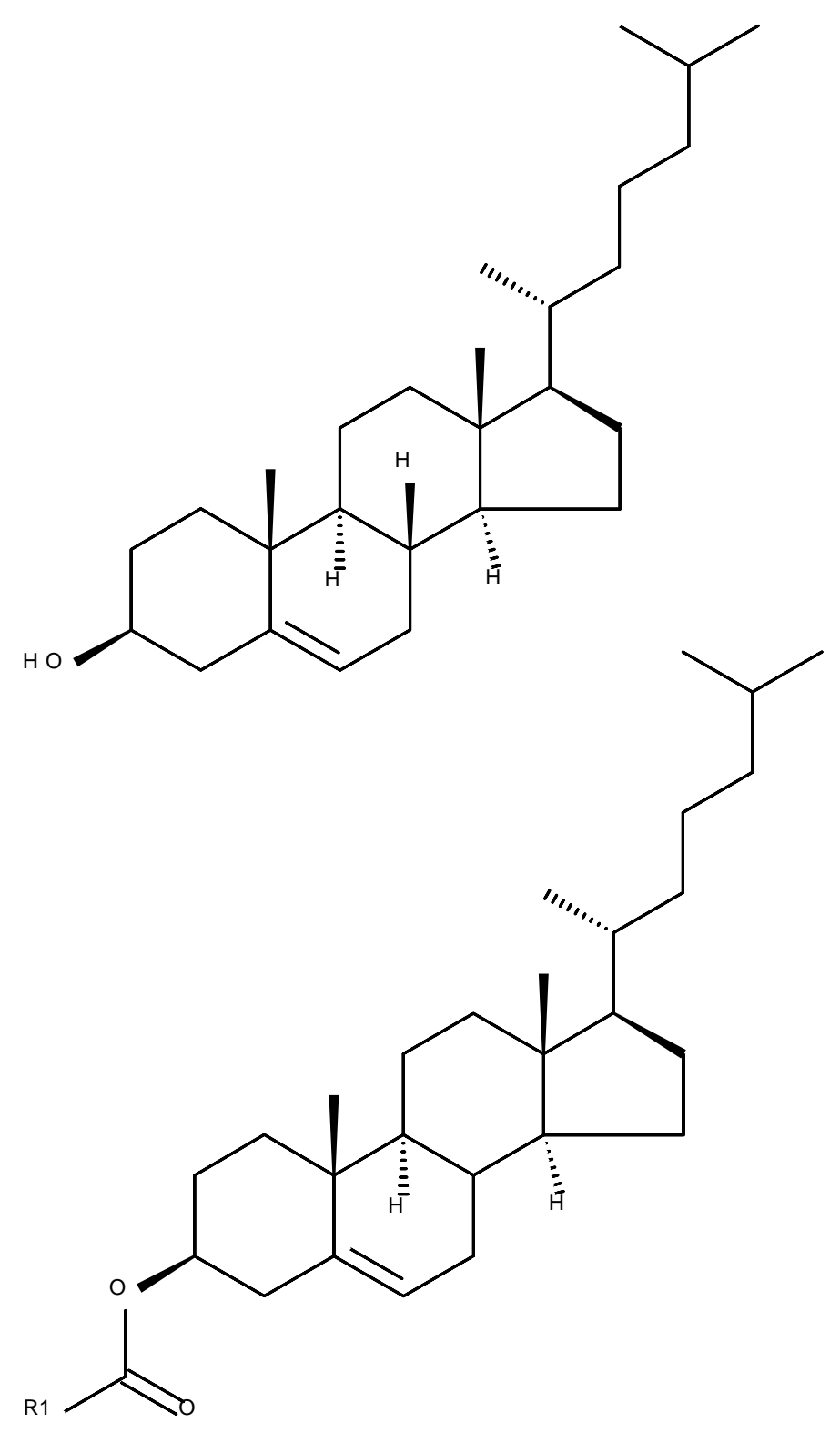


Figure 2

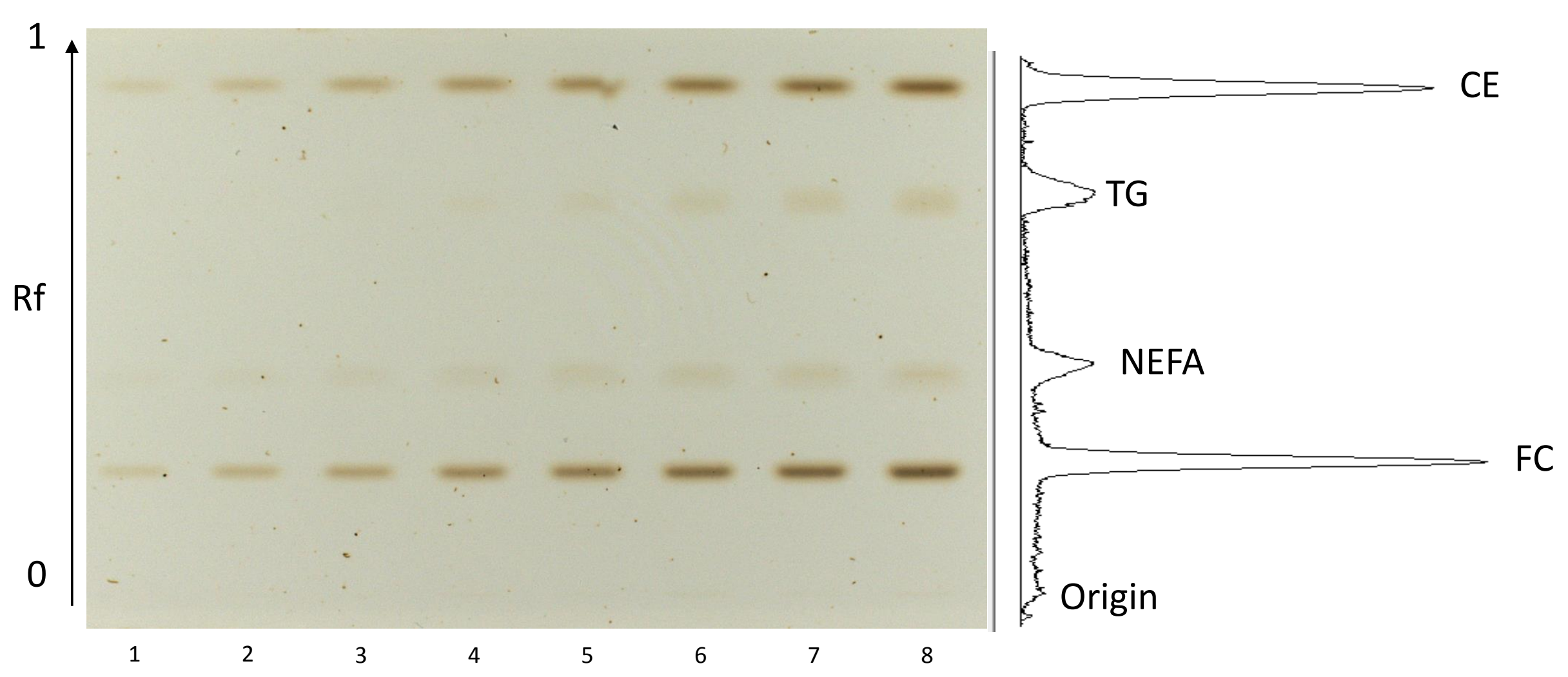


Figure 3

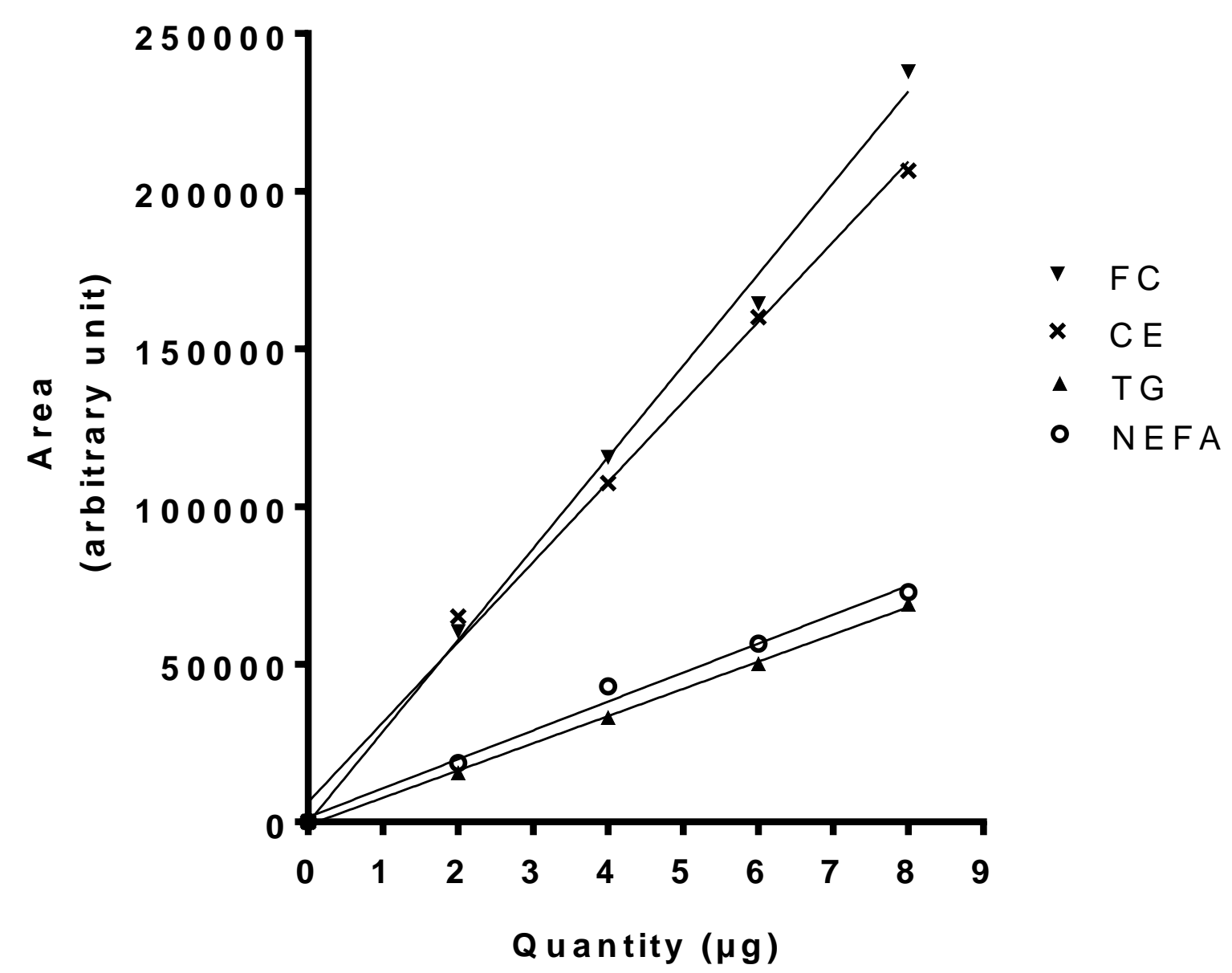


Figure 4

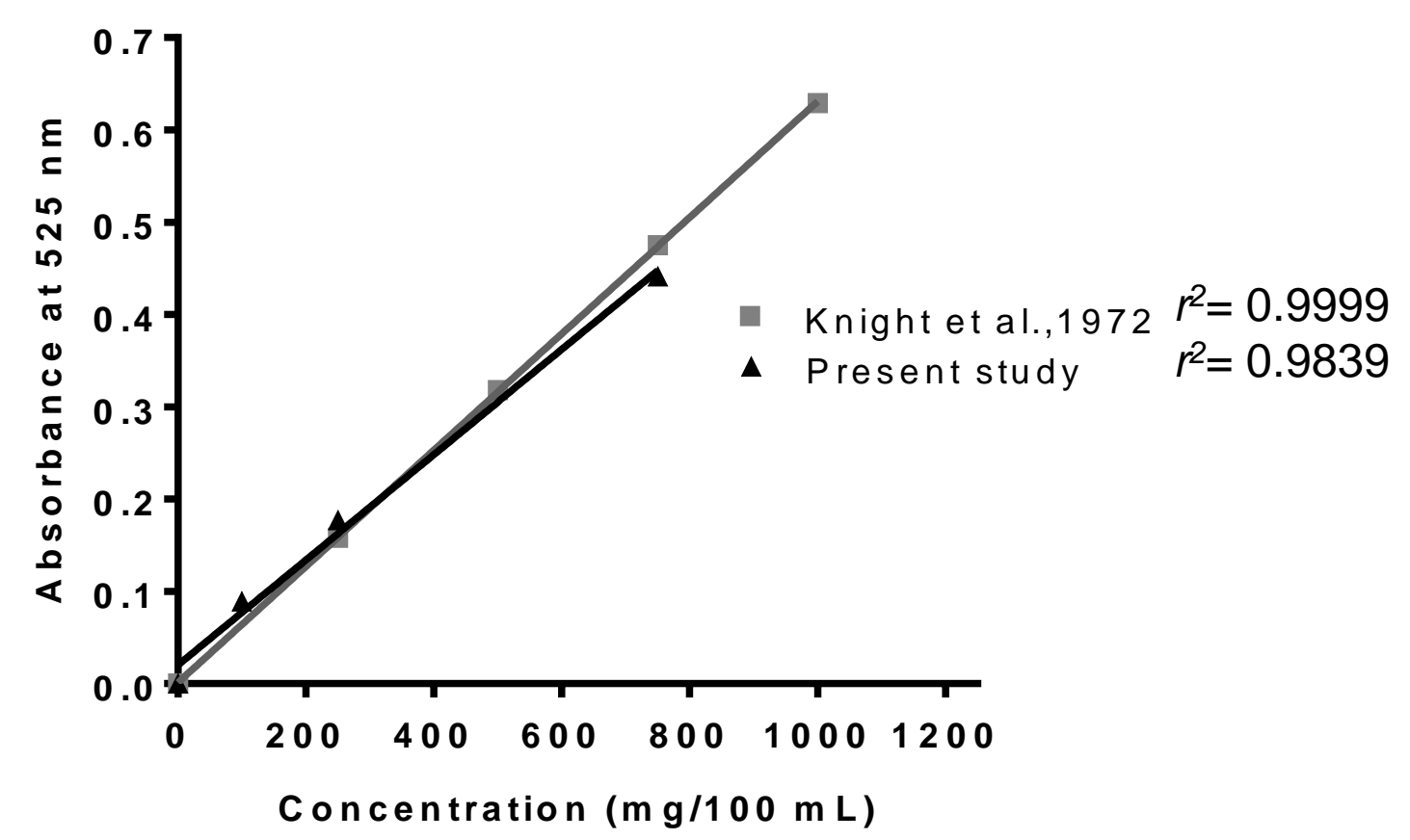


Figure 5

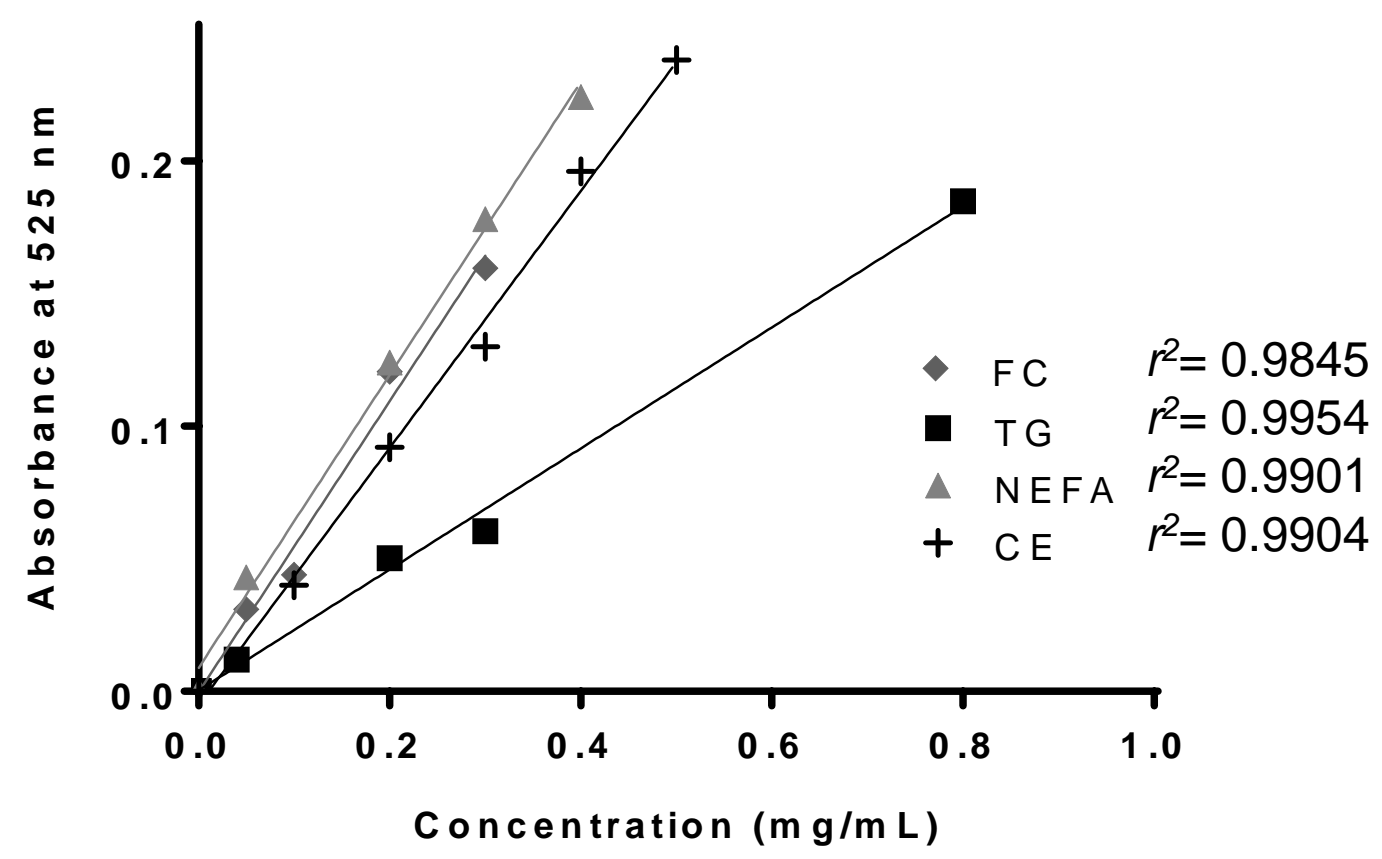


Figure 6

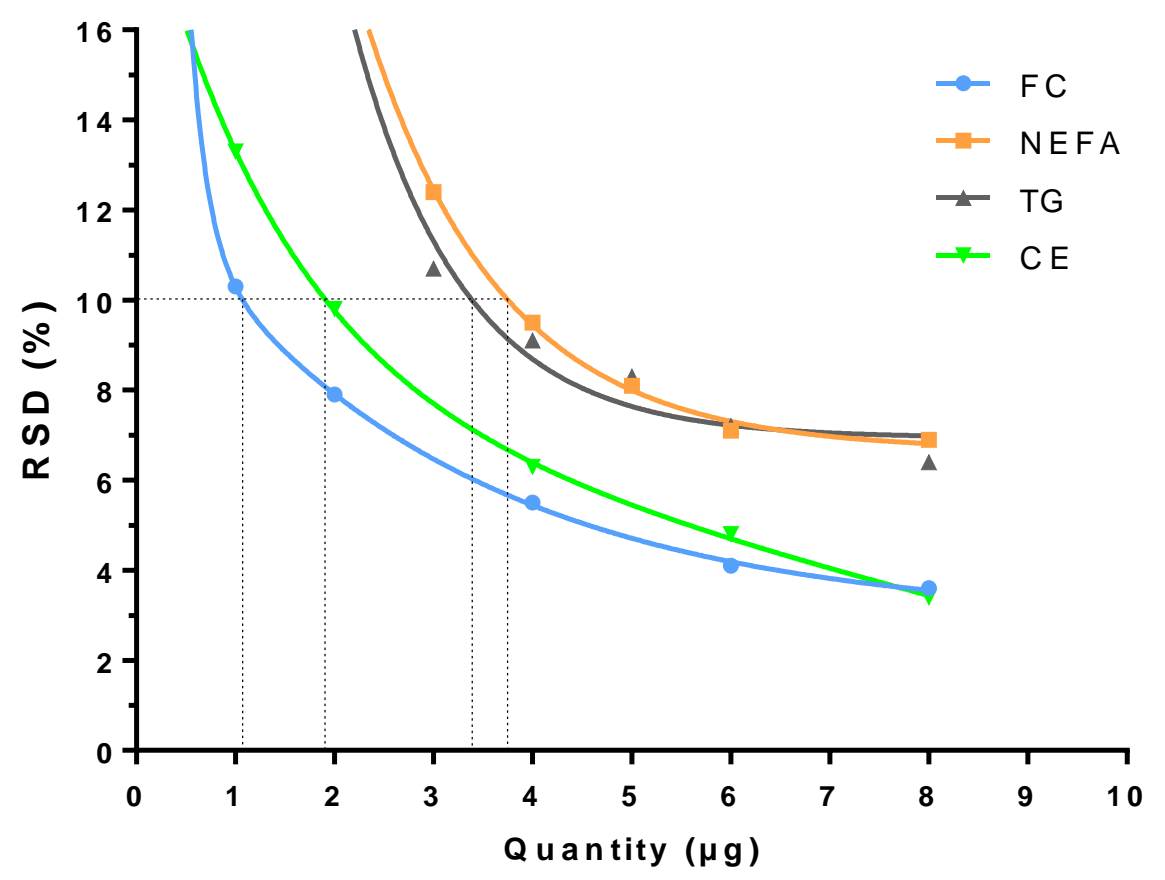


Figure 7

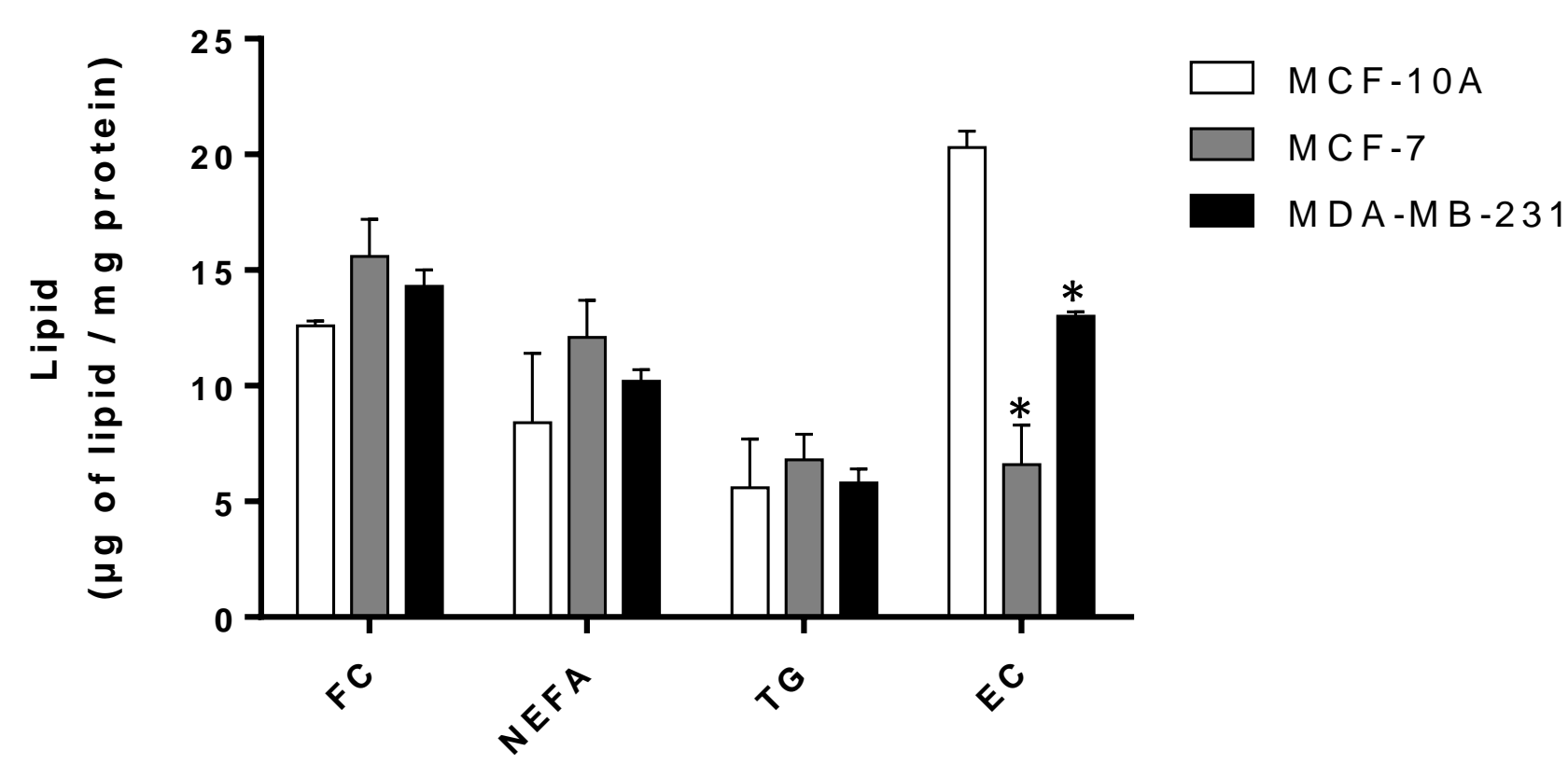


Figure 8

MCF-10A

MCF-7

MDA-MB231
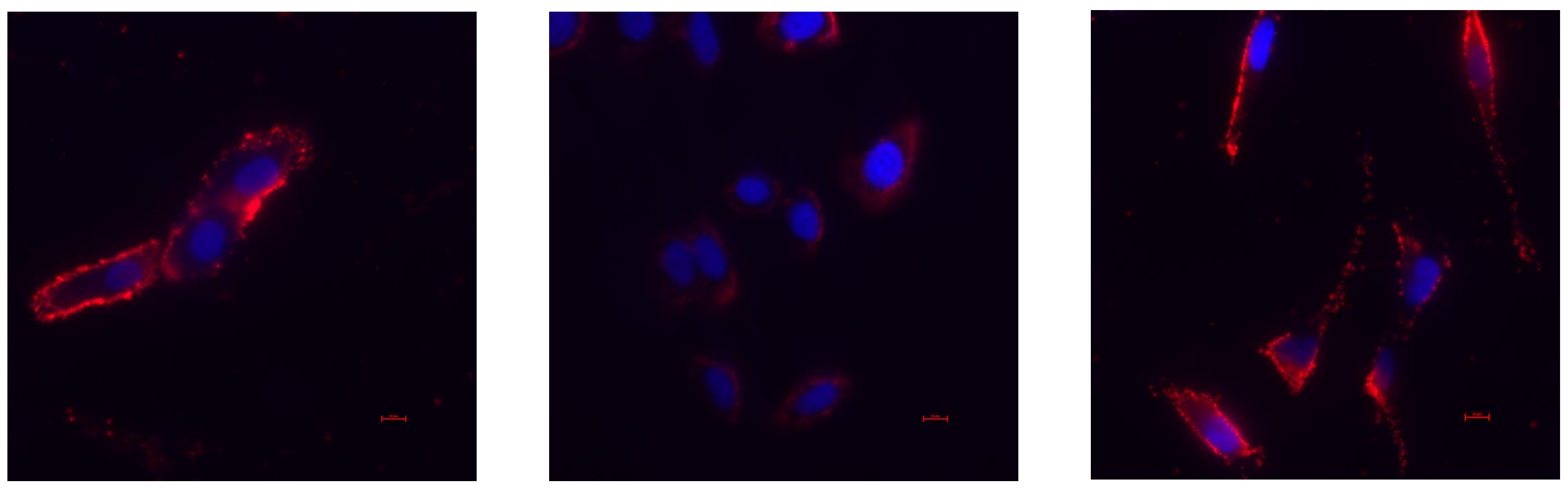